\title{
Mycodiversity of xylophilous basidiomycetes (Basidiomycota, Fungi) in Mondaí, Santa Catarina, Brazil II: A new addition
}

\author{
Marisa de Campos-Santana* \\ Clarice Loguercio-Leite \\ Laboratório de Micologia, Departamento de Botânica \\ Centro de Ciências Biológicas, Universidade Federal de Santa Catarina \\ CEP 88010-970, Florianópolis - SC, Brazil \\ *Author for correspondence \\ marisacampossantana@yahoo.com.br
}

Submetido em 25/08/2009

Aceito para publicação em 20/01/2010

\section{Resumo}

Micodiversidade de basidiomicetes (Fungi) xilófilos para Mondaí, Santa Catarina, Brasil, II: Nova contribuição. Um levantamento recente da micodiversidade de basidiomicetes xilófilos (Basidiomycota, Fungi) no município de Mondaí (Santa Catarina, Brasil) resultou na identificação de 15 espécies não registradas anteriormente para a área de estudo; todas elas são causadoras de podridão branca na madeira.

Unitermos: Basidiomycota, Floresta Estacional Decidual, micodiversidade

\section{Abstract}

In a recent survey on xylophilous basidiomycetes (Basidiomycota, Fungi) in the municipality of Mondaí (Santa Catarina, Brazil), fifteen previously unrecorded species were identified; all of them are white-rotting basidiomycetes.

Key words: Basidiomycota, Deciduous Seasonal Forest, mycodiversity

\section{Introduction}

Fungi play key roles in all ecosystems as saprophytic, pathogens and symbionts (Mueller et al., 2007; Schmit and Mueller, 2007). In spite of this, they are essential in the recycling of nature, but little is known about their population dynamics, community structure, and diversity due to difficulties encountered with the identification, isolation and quantification of many fungi (Kowalchuk, 1999).
Gilbert et al. (2002) state that despite the recognition of the megadiversity of fungi in tropical forests, few systematic studies of these groups have been done in tropical areas. While about 97,330 species of fungi have been described at global level (Kirk et al., 2008), there are not compilations of published information to enable a proper understanding of the mycodiversity and biogeographical distribution of these organisms (Schmit and Mueller, 2007). 
Moncalvo and Buchanan (2008) examined global phylogeographic relationships in the Ganoderma applanatum - australe species complex. Their conclusions, based on molecular studies, evidenced that dispersal plays a significant role in the biogeographical history of the fungi in the Southern Hemisphere. Thus the forest fragmentation is disturbing the survival of many fungi, affecting directly its strategy of dispersion and colonization. Finally, the accelerated process of environmental deterioration leads many plant species to extinction, exemplified in the dramatic reality of the Deciduous Seasonal Forest, hence the destruction of lignocellulolytic basidiomycetes associated with it.

Currently, there is little knowledge of mycodiversity and also a deficiency of systematic taxonomic work achieved in western Santa Catarina state. A single article has recorded 20 species in 8 families of basidiomycetes (= Agaricomycetes Doweld): Ganodermataceae Donk., Gloephyllaceae Jülich, Hymenochaetaceae Imazeki \& Toki, Meripilaceae Jülich, Meruliaceae Karsten, Polyporaceae Corda, Schizophyllaceae Quél. and Steccherinaceae Parmasto (Campos-Santana and Loguercio-Leite, 2008).

The aim of this work is to expand the knowledge about xilophilous basidiomycete's diversity in Mondaí, SC, Brazil.

\section{Materials and Methods}

Fungal collections were carried out between December/2005 and May/2007 at two locations (Linha Sanga Forte and Linha Uruguai) in the municipality of

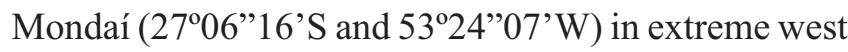
of the Santa Catarina state, southern Brazil (Figure 1). The area was originally covered by Deciduous Seasonal Forest. After collections, the basidiomata were taken to the Laboratório de Micologia (BOT/CCB/UFSC), where they were analyzed. For identification, the macroand micromorphological analyses of the specimens were obtained following the traditional methodology (Singer, 1975; Ryvarden, 1991). Voucher materials were preserved in the Herbarium FLOR (Holmgren et al., 1998). Taxonomic arrangement followed Kirk et al. (2008).

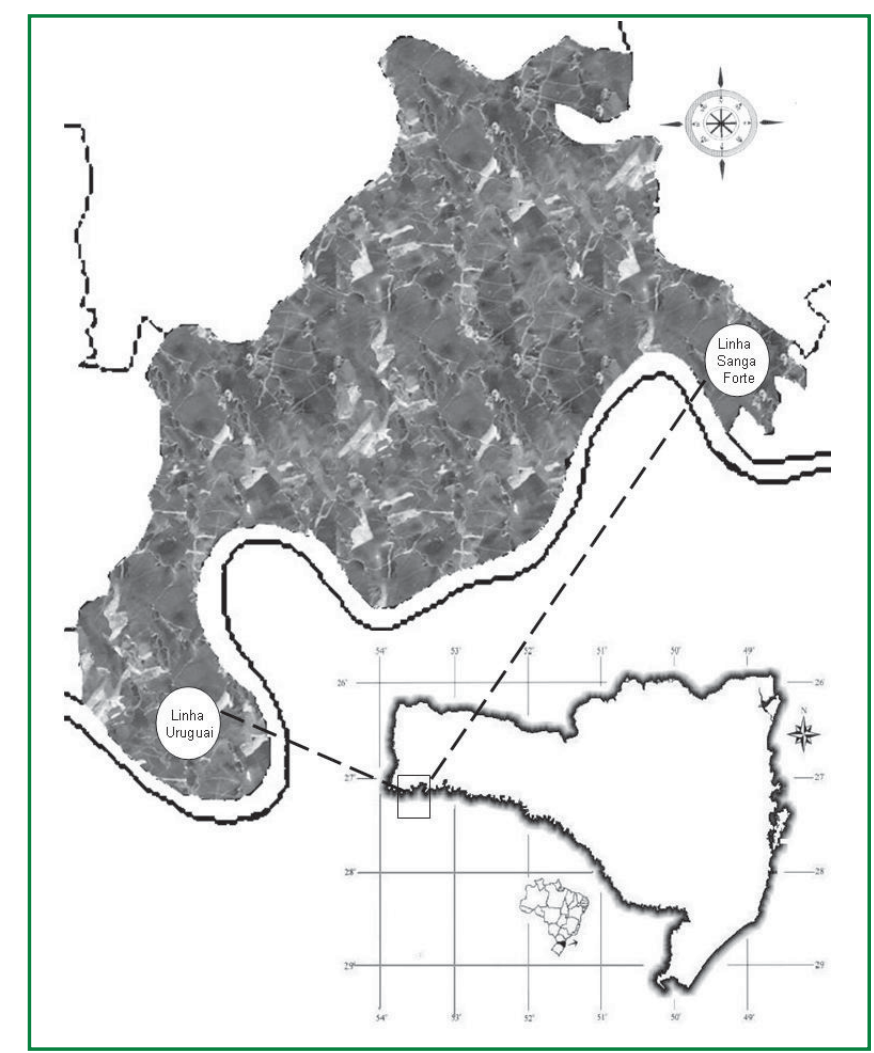

FIGURE 1: Map of Mondaí municipality showing the location of the two collection sites: Linha Sanga Forte and Linha Uruguai, Santa Catarina state, Brazil (Source: CIASC/2006. Modified by: Marisa de Campos Santana).

\section{Results and Discussion}

All species are recorded for the first time from the municipality of Mondaí. This article provides an improvement of the mycogeographical distribution of xilophilous basidiomycetes in Southern Brazil. Material examined and illustrations of hymenia and basidiospores (Figure 2) for each species are included.

HYMENOCHAETACEAE Imazeki \& Toki Bull. Govt Forest Exp. Stn Meguro 67: 24, 1954.

Fomitiporia undulata Murrill. N. Amer. Fl. 9 (1): 10, 1907.

Figure 2a

Description in Loguercio-Leite and Wright (1995).

Distribution: neotropical; Brazil (Alagoas and Santa Catarina).

Material examined: Brazil, Santa Catarina: Mondaí, Linha Uruguai, Campos-Santana \& Santana 225, 23/ V/07 (FLOR 32230). 
Phellinus allardii (Bres.) S. Ahmad, Basidiomyc.

W. Pakist. 6: 57, 1972.

$\equiv$ Fomes allardii Bres. Bull. Jard. Bot. l'État à Brux. 4: 19, 1913.

Figure $2 b$

Description in Ryvarden and Johansen (1980).

Distribution: pantropical; Brazil (Santa Catarina).

Material examined: Brazil, Santa Catarina: Mondaí, Linha Uruguai, Campos-Santana \& Santana 258, 23/ V/07 (FLOR 32241).

Phellinus glaucescens (Petch) Ryvarden, Norw. Jl. Bot. 19: 234, 1972.

$$
\begin{gathered}
\equiv \text { Poria glaucescens Petch. Ann. R. Bot. Gdns } \\
\text { Peradeniya. 6: 139, } 1916 .
\end{gathered}
$$

Figure 2c

Description in Ryvarden and Johansen (1980).

Distribution: pantropical; Brazil (Santa Catarina).

Material examined: Brazil, Santa Catarina: Mondaí, Linha Uruguai, Campos-Santana \& Santana 224, 23/ V/07 (FLOR 32242).

Phylloporia chrysita (Berk.) Ryvarden, Norw. Jl. Bot. 19: 235, 1972.

$\equiv$ Polyporus chrysites Berk., Hooker's J. Bot. Kew. Gard. Misc. 8: 233, 1856.

Figure 2d

Description in Wagner and Ryvarden (2002).

Distribution: pantropical; Brazil (Bahia, Paraná, Pará, Pernambuco, Rio Grande do Sul, Santa Catarina and São Paulo).

Material examined: Brazil, Santa Catarina: Mondaí, Linha Sanga Forte, Campos-Santana, Santana \& Zanella 115, 16/VI/06 (FLOR 32245); ibid, Linha Uruguai, Campos-Santana, Santana \& Zanella 148, 17/ VI/06 (FLOR 32246); ibid, Linha Sanga Forte, Campos-Santana \& Santana 272, 25/V/07 (FLOR 32247).

MERIPILACEAE Jülich

Biblthca Mycol. 85: 228, 1982 [1981].

Rigidoporus lineatus (Pers.) Ryvarden, Norw. Jl. Bot. 19: 236, 1972.

$\equiv$ Polyporus lineatus Pers. in Gaudichaud Bot. Frey. Voy. Monde 174, 1827.
Figure 2e

Description in Gugliotta and Bononi (1999).

Distribution: pantropical; Brazil (Alagoas, Amapá, Bahia, Paraíba, Paraná, Pernambuco, Rio Grande do Sul, Santa Catarina and São Paulo).

Material examined: Brazil, Santa Catarina: Mondaí, Linha Sanga Forte, Campos-Santana, Santana \& Rodrigues-Souza, 13, 03/I/06 (FLOR 32254); ibid, Linha Uruguai, Campos-Santana, Santana \& RodriguesSouza, 177, 27/XII/06 (FLOR 32255); ibid, Linha Sanga Forte, Campos-Santana \& Santana, 306, 25/V/07 (FLOR 32256).

Rigidoporus microporus (Fr.) Overeem, Icon. Fung. Malay. 5: 1, 1924.

$\equiv$ Polyporus microporus Fr. Syst. Mycol. 1: 376, 1821

Figure $2 \mathrm{f}$

Description in Ryvarden and Johansen (1980).

Distribution: pantropical; Brazil (Acre, Pará, Alagoas, Paraíba, Pernambuco, Amazonas, Rio Grande do Sul, Rondônia, Roraima, Santa Catarina and São Paulo).

Material examined: Brazil, Santa Catarina: Mondaí, Linha Sanga Forte, Campos-Santana \& Santana, 280, 25/V/07 (FLOR 32257).

Rigidoporus vinctus (Berk.) Ryvarden. Norw. J1. Bot. 19: 139-143, 1972.

$\equiv$ Polyporus vinctus Berk. Ann. Mag. nat. Hist. 9: 196, 1852.

Description in Ryvarden (1972).

Figure $2 \mathrm{~g}$

Distribution: pantropical; Brazil (Alagoas, Paraná, Pernambuco, Santa Catarina and São Paulo).

Material examined: Brazil, Santa Catarina: Mondaí, Linha Sanga Forte, Campos-Santana \& Santana, 285, 25/V/07 (FLOR 32260).

\section{MERULIACEAE Karsten}

Revue mycol., Toulouse 3 (9): 19, 1881.

Irpex lacteus (Fr.) Fr., Elench. fung. 1: 142, 1828.

$\equiv$ Sistotrema lacteum Fr., Observ. mycol. 2: 226, 1818.

Figure $2 \mathrm{~h}$

Description in Gilbertson and Ryvaden (1986).

Distribution: cosmopolitan; Brazil (Paraná, Pernambu- 
co, Rio Grande do Sul, Santa Catarina and São Paulo).

Material examined: Brazil, Santa Catarina: Mondaí, Linha Sanga Forte, Campos-Santana, Santana \& Souza-Rodrigues 21, 03/I/06 (FLOR 32266).

\section{Gloeoporus dichrous (Fr.) Bres.,} Hedwigia 53: 74, 1913.

$\equiv$ Polyporus dichrous Fr., Observ. Mycol. 1: 125, 1815.

Figure $2 \mathrm{i}$

Description in Núñez and Ryvarden (2001).

Distribution: cosmopolitan; Brazil (Alagoas, Amazonas, Bahia, Minas Gerais, Paraná, Pernambuco, Santa Catarina, São Paulo and Rio Grande do Sul).

Material examined: Brazil, Santa Catarina: Mondaí, Linha Uruguai, Campos-Santana \& Santana 238, 23/ V/07 (FLOR 32264); ibid, Linha Sanga Forte, ipse 300, 25/V/07 (FLOR 32265).

\section{POLYPORACEAE Corda}

Icon. Fyng. 3: 49, 1839.

Coriolopsis rigida (Berk. \& Mont.) Murrill, N. Amer. Fl. 9 (2): 75, 1908.

$\equiv$ Trametes rigida Berk. \& Mont., Annls Sci. Nat., Bot. 11: 240, 1849.

Figure $2 \mathrm{j}$

Description in Ryvarden and Johansen (1980).

Distribution: neotropical; Brazil (Alagoas, Pará, Paraíba, Paraná, Pernambuco, Rio Grande do Sul, Roraima, Santa Catarina, São Paulo and Sergipe).

Material examined: Brazil, Santa Catarina: Mondaí, Linha Sanga Forte, Campos-Santana, Santana \& Zanella 101, 16/VI/06 (FLOR 32267); ibid, Linha Uruguai, ipse 137 e 147, 17/VI/06 (FLOR 32268, FLOR 32269).

Megasporoporia setulosa (Henn.) Rajchenb., Mycotaxon 16 (1): 180, 1982.

$\equiv$ Poria setulosa Henn., Bot. Jahrb. 28: 321, 1901.

Description in Ryvarden et al. (1982).

Figure 2k

Distribution: pantropical; Brazil (Paraná and Santa Catarina).

Material examined: Brazil, Santa Catarina: Mondaí, Linha Uruguai, Campos-Santana, Santana \& Rodrigues-Souza 189, 27/XII/06 (FLOR 32271).
Perenniporia medulla-panis (Jacq.:Fr.) Donk, Persoonia 5: 76, 1967.

$\equiv$ Polyporus medulla-panis Jacq.:Fr., Syst. Mycol. 1: $380,1821$.

Figure 21

Description in Rajchenberg (2006).

Distribution: cosmopolitan; Brazil (Bahia, Paraná, Rio Grande do Sul, Santa Catarina, São Paulo and Sergipe).

Material examined: Brazil, Santa Catarina: Mondaí, Linha Sanga Forte, Campos-Santana \& Santana, 62, 25/V/07 (FLOR 32275).

Perenniporia piperis (Rick.) Rajchenb., Nordic. J1. Bot. 7 (5): 555, 1987.

三Fomes piperis Rick., Iheringia Bot. 7: 202, 1960.

Figure $2 \mathrm{~m}$

Description in Gerber et al. (1999).

Distribution: neotropical; Brazil (Rio Grande do Sul and Santa Catarina).

Material examined: Brazil, Santa Catarina: Mondaí, Linha Sanga Forte, Campos-Santana, Santana \& Zanella 122, 16/VI/06 (FLOR 32276); ibid, Linha Uruguai, Campos-Santana, Santana \& Rodrigues-Souza 170, 27/XII/06 (FLOR 32277).

Trichaptum sector (Ehrenb.) Kreisel, Monog., Cien., Univ. de Habana 16: 84, 1971.

$\equiv$ Boletus sector Ehrenb., Horae Phys. Berol. 10, 1820.

Figure 2n

Description in Gilbertson and Ryvarden (1986).

Distribution: neotropical; Brazil (Alagoas, Pará, Paraíba, Paraná, Rio Grande do Sul, Rio de Janeiro, Pernambuco, Santa Catarina and São Paulo).

Material examined: Brazil, Santa Catarina: Mondaí, Linha Sanga Forte, Campos-Santana \& Santana 61, 15/ IV/2006 (FLOR 32300).

STECCHERINACEAE Parmasto.

Consp. System. Corticiac.: 169, 1968.

Steccherinum reniforme (Berk. \& M. A. Curtis) Banker, Mem. Torrey Bot. Club. 12: 127, 1906. 
$\equiv$ Hydnum reniforme Berk. \& M.A. Curtis, J. Linn. Soc. Bot. 10: 325, 1868.

Description in Maas Geesteranus (1974).

Distribution: neotropical; Brazil (Goiás, Mato Grosso, Rio Grande do Sul, Rio de Janeiro, Santa Catarina and São Paulo).
Material examined: Brazil, Santa Catarina: Mondaí, Linha Sanga Forte, Campos-Santana, Santana \& Souza-Rodrigues 11, 03/I/06 (FLOR 32301); ibid, ipse 14, 03/I/06 (FLOR 32302); ibid, ipse 40, 03/I/06 (FLOR 32303); ibid, Campos-Santana \& Santana 52, 15/IV/06 (FLOR 32304); ibid, Campos-Santana, Santana \& Zanella 117, 16/VI/06 (FLOR 32305).
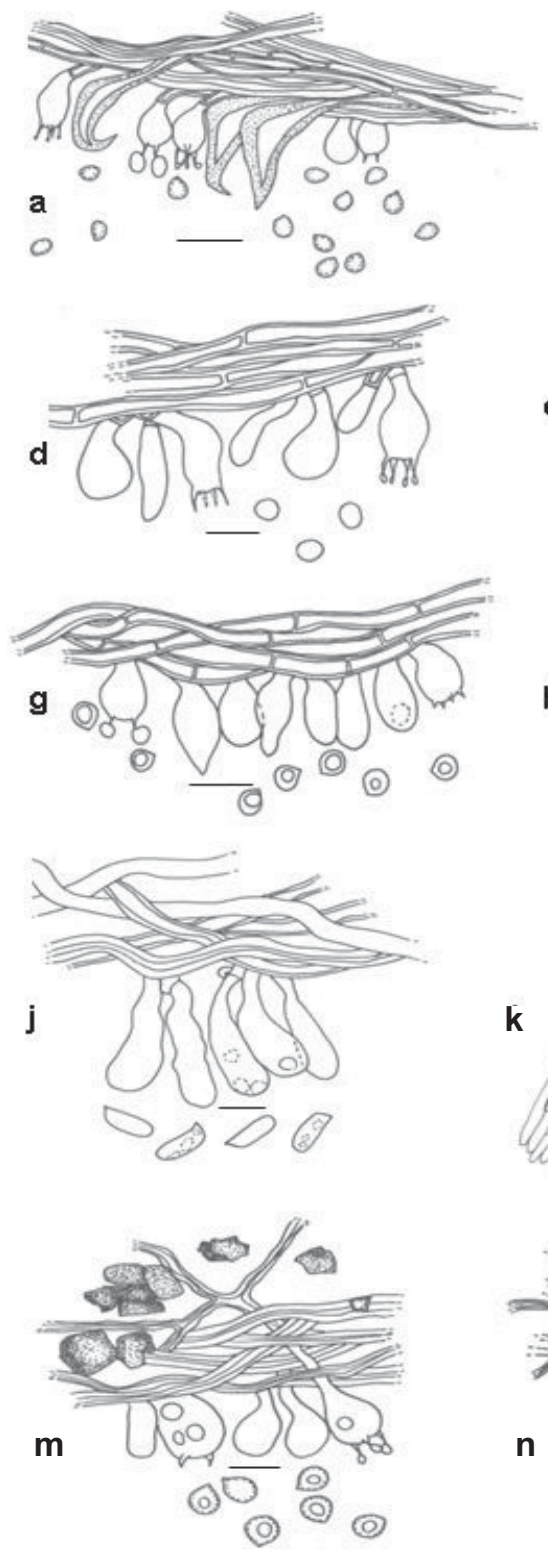
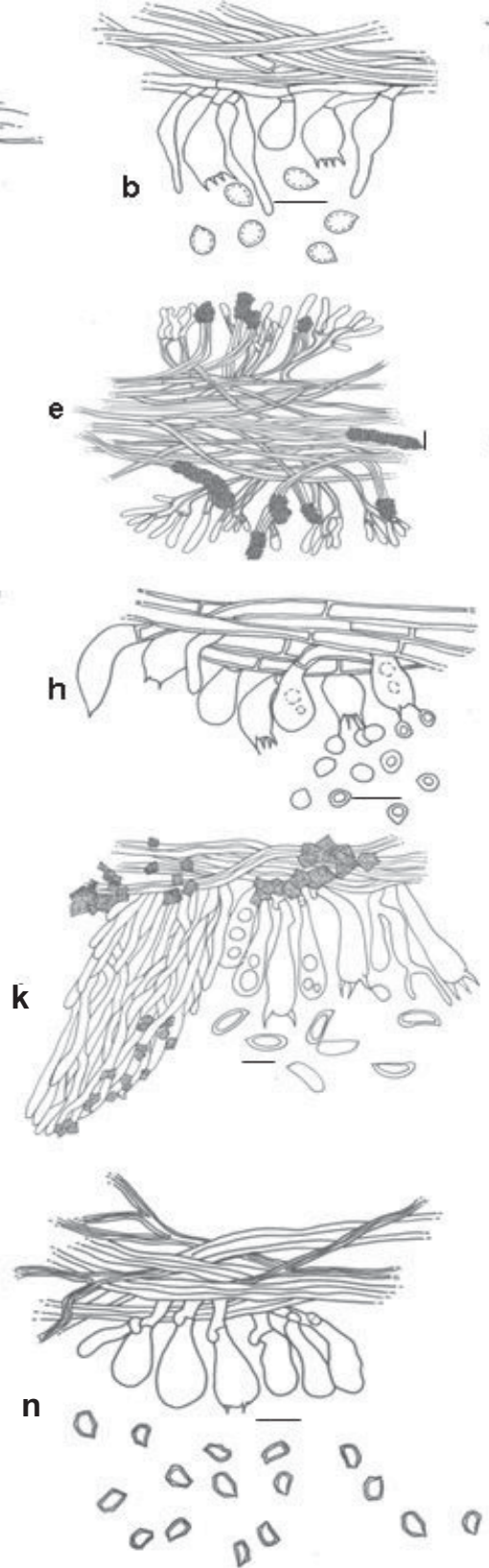
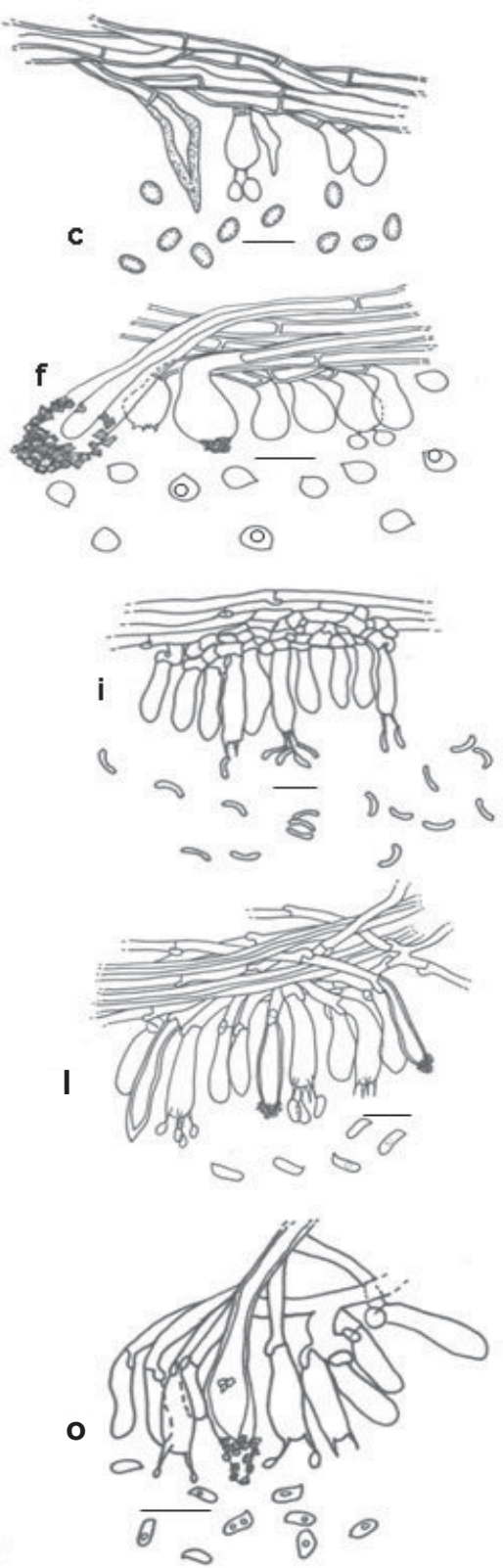

FIGURE 2: Hymenium and spores of species: a) Fomitiporia undulata, b) Phellinus allardii, c) Phellinus glaucescens, d) Phylloporia chrysita, e) Irpex lacteus, f) Rigidoporus lineatus, g) Rigidoporus microporus, h) Rigidoporus vinctus, i) Gloeoporus dichrous, j) Coriolopsis rigida, k) Megasporoporia setulosa, 1) Trichaptum secto, m) Perenniporia medulla-panis, n) Perenniporia piperis and o) Steccherinum reniforme. Bar $=10 \mu \mathrm{m}$. 
This work added 15 species to the mycological diversity of Mondaí, increasing the number of species recorded to 35 . The thirty-five species studied from the municipality of Mondaí exhibited different geographical distribution (cosmopolitan or tropical). Most of the reported species showed a tropical distribution: 8 neotropical (almost 22.85\%) and 18 pantropical species $(51.44 \%)$. Only 9 are considered widely cosmopolitan $(25.71 \%)$. The results revealed a high mycodiversity in Deciduous Seasonal Forest. Considering the neotropical species, Stiptophyllum erubescens (Berk.) Ryvarden and Mycobonia flava (Sw.) Fr. were collected only in this type of forest. It must be emphasize that one species (Rigidoporus amazonicus Ryvarden) is only known to Brazil. Considering the type of wood rotting only one (Stiptophyllum erubescens) is brow rot.

\section{Acknowledgements}

We express our sincere gratitude to the reviewers for their valuable comments and taxonomic advice.

\section{References}

Campos-Santana, M.; Loguercio-Leite, C. 2008. First records of xylophilous Basidiomycetes (Fungi) in Mondaí, Santa Catarina, Southern Brazil. Biotemas, 21 (2): 19-26.

Gerber, A. L.; Neves, M. A.; Loguercio-Leite, C. 1999. Some species of Perenniporia Murrill (Poliales, Basidiomycotina) from Southern Brazil. Revista Brasileira de Botânica, 22 (2): 185-193. Gilbert, G. S.; Ferrer, A.; Carranza, J. 2002. Polypore fungal diversity and host density in a moist tropical forest. Biodiversity and Conservation, 11: 947-957.

Gilbertson, R. L.; Ryvarden, L. 1986. North America Polypores. Abortiporus - Lindtneria. v.1. Fungi Flora, Oslo, Norway, 433pp. Gugliotta, A. M.; Bononi, V. L. R. 1999. Polyporaceae do Parque Estadual da Ilha do Cardoso, São Paulo, Brasil. Boletim do Instituto de Botânica, 12: 1-112.

Holmgren, P. K.; Holmgren, N. H.; Barnett, L. C. 1998. Index Herbariorum. New York Botanical Garden, New York, USA. Available at $<\mathrm{http} / / /$ sciweb.nybg.org/science2/IndexHerbariorum. asp $>$. Accessed on February $2^{\text {nd }} 2009$.
Kirk, P. M.; Cannon, P. F.; David, J. C.; Stalpers, J. 2008. Ainsworth \& Bisby's Dictionary of the Fungi. $10^{\text {th }}$ ed. CAB International, Wallingford, UK, 771pp.

Kowalchuk, G. A. 1999. New perspectives towards analysing fungal communities in terrestrial environments. Current Opinion in Biotechnology, 10: 247-251.

Loguercio-Leite, C.; Wright, J. E. 1995. The genus Phellinus (Hymenochaetaceae) on the Island of Santa Catarina, Brazil. Mycotaxon, 51: 361-388.

Maas Geesteranus, R. A. 1974. Studies in the Genera Irpex and Steccherinum. Personia, 7 (4): 443-581.

Moncalvo, J. M.; Buchanan, P. K. 2008. Molecular evidence for long distance dispersal across the Southern Hemisphere in the Ganodermaapplanatum-australe species complex (Basidiomycota). Mycological Research, 112: 425-436.

Mueller, G. M.; Schmit, J. P.; Leacock, P. R.; Buyck, B.; Cifuentes, J.; Desjardin, D. E.; Halling, R. E.; Hjortstam, K.; Iturriaga, T.; Larsson, K-H.; Lodge, D. J.; May, T. W.; Minter, D.; Rajchenberg, M.; Redhead, S. A.; Ryvarden, L.; Trappe, J. M.; Watling, R.; $\mathrm{Wu}$, Q. 2007. Global diversity and distribution of macrofungi. Biodiversity and Conservation, 16: 37-48.

Núñez, M.; Ryvarden, L. 2001. East Asia Polypores 2. Polyporaceae sensu lato. Synopsis Fungorum, 14: 169-522.

Rajchenberg, M. 2006. Los Políporos (Basidiomycetes) de los Bosques Andinos Patagónicos de Argentina. Bibliotheca Mycologica, 201: 1-300.

Ryvarden, L. 1972. The genera Stereum (sensu lato) and Hymenochaete in Norway. Norwegian Journal of Botany, 18: 97-108.

Ryvarden, L. 1991. Genera of Polypores - Nomenclature and taxonomy. Synopsis Fungorum, 5: 1-363.

Ryvarden, L.; Johansen, I. 1980. A preliminary polypore flora of East Africa. Fungiflora, Oslo, Norway, 636pp.

Ryvarden, L.; Wright, J. E.; Rajchenberg, M. 1982. Megasporoporia a new genus of resupinate Polypores. Mycotaxon, 16, (1): 172182.

Schmit, J. P.; Mueller, G. M. 2007. An estimate of lower limit of global fungal diversity. Biodiversity and Conservation, 16: 99111.

Singer, R. 1 The Agaricales in modern taxonomy. $3^{\text {rd }}$ ed. Vaduz. J. Cramer, Stuttgart, Germany, 912pp.

Wagner, T.; Ryvarden, L. 2002. Phylogeny and taxonomy of the genus Phylloporia (Hymenochaetales). Mycological Progress, 1 (1): 105-116. 